\title{
First-principles calculations and thermodynamic modeling of the Sn-Ta system
}

\author{
Cassie Marker ${ }^{*}$, ShunLi Shang, Xuan L. Liu, Greta Lindwall ${ }^{1}$, Zi-Kui Liu \\ Department of Materials Science and Engineering, The Pennsylvania State University, \\ University Park, PA, 16802, USA \\ *cassie.marker@gmail.com
}

\begin{abstract}
A thermodynamic description of the Sn-Ta system was developed using the CALPHAD (CALculation of PHAse Diagram) approach in combination with first-principles calculations. A positive enthalpy of mixing of the body centered cubic phase was predicted, using special quasirandom structures (SQS), indicating the tendency to form a miscibility gap. The finite temperature thermodynamic properties of $\mathrm{Ta}_{3} \mathrm{Sn}$ and $\mathrm{TaSn}_{2}$ were calculated by the DebyeGrüneisen model as well as phonon calculations using the supercell approach. The results from first-principles calculations along with the previously reported decomposition temperature of $\mathrm{TaSn}_{2}, 868 \mathrm{~K}$, were used to evaluate the Gibbs energy parameters of the $\mathrm{TaSn}_{2}, \mathrm{Ta}_{3} \mathrm{Sn}$ intermetallics and the liquid phase. No decomposition temperature of $\mathrm{Ta}_{3} \mathrm{Sn}$ has previously been reported in the literature but was predicted in this work to be $2884 \mathrm{~K}$. The calculated phase diagram agrees well with available experimental information of the Sn-Ta system and also compares well with the similar systems such as $\mathrm{Nb}-\mathrm{Sn}$ and $\mathrm{V}-\mathrm{Sn}$.
\end{abstract}

Keywords: Tin (Sn), Tantalum (Ta), special quasi-random structures (SQS), density functional theory (DFT), CALculation of PHAse Diagrams (CALPHAD)

\footnotetext{
1 Present address: National Institute of Standards and Technology, Gaithersburg, MD 20899
} 


\section{Introduction}

In addition to extensive aerospace applications, titanium ( $\mathrm{Ti}$ ) alloys are being used increasingly in medical applications, due mainly to their mechanical properties and biocompatibility. For applications, such as load-bearing implants, one of the leading issues is finding a biocompatible alloy with a Young's modulus closely matching that of bone [1]. The current load-bearing implant materials such as stainless steels, Ti-6Al-4V, and CoCrMo alloys have a higher elastic modulus (normally between 110-230 GPa) compared to that of bone (10-40 GPa). This mismatch results in stress shielding so that the implant absorbs most of the stress to the joint leading to bone death and implant failure [1,2]. To be able to achieve materials with a lower elastic modulus, efforts have been aimed at investigating more complex multi-component systems of Ti.

Currently, the biomaterial implant research of Ti alloys is focused on biocompatible elements that stabilize the body centered cubic (bcc, $\beta$ ) phase of Ti and help to lower its elastic modulus. Tantalum (Ta) is a biocompatible element and is considered to be a strong $\beta$-stabilizers [3]. Recently, tin ( $\mathrm{Sn}$ ) has also been researched for use in Ti-alloys due to its biocompatibility and low cost [4]. Kuroba et al. [5] studied various Ti-alloys such as Ti-29-Nb-13Ta-2Sn (weight percentage, and similarly hereinafter unless specified otherwise), Ti-29Nb-13Ta-4Mo, and Ti29Nb-13Ta-6Sn for use as biocompatible implant materials. Kuroba and Hagiwara [6] also studied new Ti-Cu-Ni-Sn-Ta alloys for the artificial materials used in orthopedic surgeries. The Sn-Ta system is thus an important sub-system for this purpose [7]. A complete knowledge base of the thermodynamic description of Sn-Ta can be used to examine the effects of temperature and composition on phase stability for higher order systems and help to tailor experimental alloy selections to viable options. The Sn-Ta system has three solid solution phases and two intermetallic compounds, i.e. the bcc, body centered tetragonal (bct), and diamond solution phases, and the intermetallic compounds $\mathrm{Ta}_{3} \mathrm{Sn}$ with space group $P \mathrm{~m} \overline{3} \mathrm{n}$ and $\mathrm{TaSn}_{2}\left(\mathrm{Ta}_{1.2} \mathrm{Sn}_{1.8}\right)$ with space group $F d d d$ [8].

The CALculation of PHAse Diagram (CALPHAD) technique, in combination with firstprinciples and phonon calculations based on the density functional theory (DFT), has been 
proven to provide valuable data to model the thermodynamic properties of binary and ternary systems [9]. In the present work, thermodynamic data was predicted using first-principles calculations for the two intermetallics and for the bcc, bct and diamond solution phases. The finite temperature properties of the phases were obtained using the Debye-Grüneisen model [10] and phonon calculations based on the supercell approach [11]. The DFT data was used to model the parameters of the Gibbs energy of each phase using the CALPHAD technique.

\section{Review of previous work}

The Sn-Ta binary system was studied by Okamoto [8], Studnitzky and Schmid-Fetzer [12], and Basile [13]. Both of the intermetallic phases, $\mathrm{Ta}_{3} \mathrm{Sn}$ and $\mathrm{TaSn}_{2}$, were shown to have a very narrow homogeneity range. Basile [13] observed that $\mathrm{TaSn}_{2}$ is located around $\mathrm{Ta}_{1.2} \mathrm{Sn}_{1.8}$ which was then designated as $\mathrm{Ta}_{2} \mathrm{Sn}_{3}$ by Okamoto [8]. It seems that $\mathrm{TaSn}_{2}$ is a more compatible description of the stoichiometric compound based on the descriptions of similar systems (V-Sn, and $\mathrm{Nb}-\mathrm{Sn}$ ) [14-16], and thus will be used in the present work. Basile [13] determined $\mathrm{TaSn}_{2}$ has a peritectic reaction at $595{ }^{\circ} \mathrm{C}$ and used X-ray diffraction (XRD) to elucidate the lattice parameters of $\mathrm{TaSn}_{2}$,

Studnitzky and Schmid-Fetzer [12] used powder samples to study the $\mathrm{Ta}_{3} \mathrm{Sn}$ intermetallic phase and verified the results previously reported by Basile [13]. They cold pressed the pure element powders at $600 \mathrm{MPa}$ and then heated the pellets at $1000{ }^{\circ} \mathrm{C}$ for up to 48 hours. The resulting pellet was then cold pressed at $600 \mathrm{MPa}$ again. Under these conditions $\mathrm{TaSn}_{2}$ was observed at $400{ }^{\circ} \mathrm{C}$, but was not present as the temperature increased to $600{ }^{\circ} \mathrm{C}$. In the work by Courtney et al. [17], $\mathrm{Ta}_{3} \mathrm{Sn}$ was studied to see how the temperature affects the long-range ordering parameter. In Courtney et al.'s work, $\mathrm{Ta}_{3} \mathrm{Sn}$ powder samples were sintered at 600, 700, 950, 1200, and 1450 ${ }^{\circ} \mathrm{C}$ for 2, 4, 7, and 16 days, respectively. Each sample was then studied using XRD at room temperature to examine the phases present and the long-range ordering. They concluded that the transition temperature of superconductivity for $\mathrm{Ta}_{3} \mathrm{Sn}$ varied by a maximum of $4 \mathrm{~K}$ based on heat treatment and sintering times due to long-range ordering that occurred. Courtney et al. also measured the lattice parameter of each sample and reported the average value of this cubic phase being $5.285 \AA$. 


\section{Methodologies}

\subsection{First-principles thermodynamics}

DFT-based first-principles calculations can be used to predict the Helmholtz energy, $F(V, T)$ as a function of temperature $T$ and volume $V$ via the quasiharmonic approach [10,18]:

Eq. 1

$$
F(V, T)=E_{0}(V)+F_{v i b}(V, T)+F_{T-e l}(V, T)
$$

where $\mathrm{E}_{0}$ is the static contribution at $0 \mathrm{~K}$ without the contribution of zero-point vibrational energy, $F_{V i b}$ the temperature-dependent vibrational contribution, and $F_{T-e l}$ the thermal electronic contribution. At ambient pressure, the Helmholtz energy of the system is equal to the Gibbs energy, which is used in the CALPHAD modeling. In the present work, the $E_{0}$ was calculated from the equation of state (EOS) fitted to the first-principles data points using the four-parameter Birch-Murnaghan (BM4) EOS [10]:

Eq. 2

$$
E_{0}(V)=a+b V^{-2 / 3}+c V^{-4 / 3}+V^{-2}
$$

The EOS fitting is achieved through an energy-volume (E-V) curve of seven different volumes based on the methodology discussed in details by Shang et al. [10]. The thermal electronic contribution was based on the electronic density of states and calculated with the Fermi-Dirac statistics [18]. The vibrational contribution can be obtained through the phonon quasiharmonic supercell approach or the Debye-Grüneisen method. The phonon supercell approach is a more accurate approach compared to the Debye model but it is also more computationally expensive. In the present work, both the phonon and Debye models were used for comparison. The vibrational contribution was obtained through phonon calculations at different volumes (five used herein) [18]:

Eq. 3

$$
F_{v i b}(V, T)=k_{B} T \int_{0}^{\infty} \ln \left[2 \sinh \frac{\hbar \omega}{2 k_{B} T}\right] g(\omega) d \omega
$$

where $g(\omega)$ is the phonon density of states as a function of phonon frequency $\omega$ at volume V. In addition, the Debye-Grüneisen model was used to estimate vibrational contribution [10]: 
Eq. $4 \quad F_{\text {vib }}(V, T)=\frac{9}{8} k_{B} \theta_{D}(V)-k_{B} T\left[D\left(\frac{\theta_{D}(V)}{T}\right)+3 \ln \left(1-e^{-\theta_{D}(V) / T}\right)\right]$

where $\theta_{D}$ is the Debye temperature, $T$ the temperature, and $D\left[\theta_{D}(V) / T\right]$ the Debye function. The Debye temperature can be calculated through:

Eq. 5

$$
\theta_{D}=s \frac{\left(6 \pi^{2}\right)^{1 / 3} h^{1 / 6}}{k B} V_{0}^{1 / 6}\left(\frac{B_{0}}{M}\right)^{1 / 2}\left(\frac{V_{0}}{V}\right)^{Y}
$$

where $s$ is the Debye temperature scaling factor, $\gamma$ the Grüneisen parameter determined by $B$ ' the pressure derivative of bulk modulus, $B_{0}$ the equilibrium bulk modulus, $M$ atomic mass, and $V_{O}$ equilibrium volume. Here, the equilibrium properties $V_{0}, B_{0}$, and $B$ ' are estimated from the EOS of Eq. (2). Moruzzi et al. [19] estimated the scaling factor to be 0.617 for nonmagnetic metals, but this value has been shown to be less accurate for other materials. For the present work, the scaling factor of $\mathrm{TaSn}_{2}$ and $\mathrm{Ta}_{3} \mathrm{Sn}$ were calculated from the Poisson's ratio [20],

Eq. 6

$$
s(v)=3^{5 / 6}\left[4 \sqrt{2}\left(\frac{1+v}{1-2 v}\right)^{3 / 2}+\left(\frac{1+v}{1-v}\right)^{3 / 2}\right]^{-1 / 3}
$$

where $v$ is the Poisson's ratio, which can be calculated from the elastic stiffness constants in terms of the Hill approximation [21]. The elastic stiffness constants were determined by applying $\mathrm{a} \pm 0.01$ strain to the crystal lattice and the set of stresses are determined with first-principles calculations [22]. The elastic stiffness constants are then determined from Hooke's law as follows:

Eq. $7 \quad\left(\begin{array}{lll}\varepsilon_{1,1} & & \varepsilon_{1, n} \\ \varepsilon_{2,1} & & \varepsilon_{2, n} \\ \varepsilon_{3,1} & & \varepsilon_{3, n} \\ \varepsilon_{4,1} & \cdots & \varepsilon_{4, n} \\ \varepsilon_{5,1} & & \varepsilon_{5, n} \\ \varepsilon_{6,1} & & \varepsilon_{6, n}\end{array}\right)^{-1}\left(\begin{array}{ccccccc}\sigma_{1,1} & & \sigma_{1, n} \\ \sigma_{2,1} & & \sigma_{2, n} \\ \sigma_{3,1} & & \sigma_{3, n} \\ \sigma_{4,1} & \cdots & \sigma_{4, n} \\ \sigma_{5,1} & & \sigma_{5, n} \\ \sigma_{6,1} & & \sigma_{6, n}\end{array}\right)=\left(\begin{array}{cccccc}c_{11} & c_{12} & c_{13} & 0 & 0 & 0 \\ c_{21} & c_{22} & c_{23} & 0 & 0 & 0 \\ c_{31} & c_{32} & c_{33} & 0 & 0 & 0 \\ 0 & 0 & 0 & c_{44} & 0 & 0 \\ 0 & 0 & 0 & 0 & c_{55} & 0 \\ 0 & 0 & 0 & 0 & 0 & c_{66}\end{array}\right)$

To calculate the enthalpy of formation of the bcc phase across the entire composition range, the enthalpy of formation of $\mathrm{Ta}$ and $\mathrm{Sn}$ in the bcc phase were calculated with five different compositions of $\mathrm{Ta}_{1-\mathrm{x}} \mathrm{Sn}_{\mathrm{x}}$, where $\mathrm{x}=0.0185$ ( $\mathrm{Ta}_{53} \mathrm{Sn}, 54$ atoms), 0.25, 0.5, 0.75, and 0.9815 $\left(\mathrm{TaSn}_{53}, 54\right.$ atoms). For $\mathrm{x}=0.0185$ and 0.9815 , calculations were performed on a diluted 54 atom cell where all atoms but one was $\mathrm{Sn}$ or $\mathrm{Ta}\left(\mathrm{Ta}_{53} \mathrm{Sn}\right.$ and $\left.\mathrm{TaSn}_{53}\right)$. For $\mathrm{x}=0.25,0.5$, and $0.75,16$ - 
atom special quasirandom structures (SQS) in the bcc phase developed by Jiang et al. [23] were used to mimic the behavior of random structures. The relaxation of these structures is complicated because the local atomic relaxations can cause the structure to lose the bcc lattice symmetry. To preserve the lattice symmetry, the calculations were carried out with different relaxation schemes to determine the lowest energy while retaining the bcc lattice symmetry. The structures were first relaxed by constraining everything but the cell volume. Then the structure was relaxed again constraining everything but the cell volume and shape. Finally, the structure shape, ions and cell volume were allowed to relax. The symmetry and energy were compared for each step and the structure with the lowest energy that retained symmetry was used for each composition. The selected structure was then spilt into five volumes and a static calculation and EOS fitting were performed. The enthalpy of formation was plotted as a function of composition and then used for the modeling.

In the present work, the Vienna ab-initio Simulation Package (VASP) was used to perform the first-principles calculations [24]. The projector augmented-wave (PAW) [25,26] method was used to describe the electron-ion interactions with exchange correlation functional being elucidated by the generalized gradient approximation (GGA-PBE) as implemented by Perdew, Burke, and Ernzerhof [27]. A sigma value of 0.2 and a plane wave energy cutoff of 1.3 times higher than the highest default cutoff was adopted. The Brillouin zone sampling was done with Blöchl corrections [26] using a gamma centered Monkhorst-Pack (MP) scheme [28]. The kpoints grid for diamond-Sn, bcc-Ta, TaSn 2 , and bcc-Sn were 4x4x4, 6x6x6, 10x10x5, and 6x6x6 respectively. The k-point grids for the bct-Sn, $\mathrm{Ta}_{3} \mathrm{Sn}$ and bcc SQS calculations used an automated k-point mesh generator in VASP with the length of the subdivisions specified as 80.

The energy convergence criterion of the electronic self-consistency is set as $10^{-4} \mathrm{eV} /$ atom for all of the calculations. The Sn was treated with the d electrons as valance, and the Ta was treated with the p electrons as valance.

\subsection{CALPHAD modeling}

CALPHAD modeling was used to evaluate the parameters of the Gibbs energy function for each individual phase [9]. The Gibbs energy functions of pure elements were adopted from the SGTE (SSUB) database [29]. In the present work, the bcc and liquid phases were modeled in 
conjunction with the two intermetallics $\mathrm{Ta}_{3} \mathrm{Sn}$ and $\mathrm{TaSn}_{2}$. Dilute first-principles calculations of $\mathrm{Ta}$ in Sn were done for the diamond and bet phases. However, there is little solubility of Ta in these phases and there is no description of pure Ta in these phases available in SGTE. So no binary interaction parameters were introduced in the modeling similar to other Sn systems such as $\mathrm{Nb}-\mathrm{Sn}$ and $\mathrm{V}-\mathrm{Sn}[16,14]$. In the present work, the energy of the liquid and bcc solution phases were modeled as:

Eq. $8 \quad G_{m}^{\phi}=x_{T a}{ }^{o} G_{T a}^{\phi}+x_{S n}{ }^{o} G_{S n}^{\phi}+R T\left[x_{T a} \ln \left(x_{T a}\right)+x_{S n} \ln \left(x_{S n}\right)\right]+{ }^{X S} G_{m}^{\phi}$

where $x_{\mathrm{Ta}}$ and $x_{\mathrm{Sn}}$ are the mole fractions of Ta and Sn, respectively. ${ }^{0} G_{i}^{\phi}$ is the molar Gibbs energy of pure element, $i$, in the specific phase being modeled taken from the SGTE database [29]. The last term represents the excess mixing energy, ${ }^{X S} G_{m}^{\phi}$, expressed as [30]:

Eq. 9

$$
{ }^{X S} G_{m}^{\phi}=x_{T a} x_{S n} \sum_{j=0}{ }^{j} L_{T a, S n}^{\phi}\left(x_{T a}-x_{S n}\right)^{j}
$$

Here, ${ }^{j} L_{T a, S n}^{\phi}$ is the interaction parameter and can be defined as:

Eq. 10

$$
{ }^{j} L_{T a, S n}^{\phi}={ }^{j} a+{ }^{j} b T
$$

where $a$ and $b$ are parameters being modeled.

For the intermetallics, the Gibbs energy in per mole unit formula used in the present work is of the form [31]:

Eq. 11

$$
{ }^{o} G_{m}^{T a_{x} S n_{y}}=a+b T+x^{*}{ }^{o} G_{T a}^{b c c}+y^{*}{ }^{o} G_{S n}^{b c t}
$$

where $a$ and $b$ are model parameters determined from the enthalpy of formation and the heat capacity. 
The data from the first-principles calculations were accounted for in the parameter optimization together with experimental information using the PARROT module in Thermo-Calc software [32].

\section{Results and discussion}

\subsection{First-principle calculations}

To evaluate the accuracy of phonon calculations for the present system, both the dispersion curves and the phonon density of states (DOS) are plotted for bcc-Ta, bct-Sn, $\mathrm{TaSn}_{2}$, and $\mathrm{Ta}_{3} \mathrm{Sn}$ in Figure 1, Figure 2, Figure 3, and Figure 4, respectively. The bcc-Ta phonon dispersion curve in Figure 1 is compared with values obtained by Taioli et al. [33] using neutron scattering, showing good agreement. The longitudinal modes (LO) and the transverse modes (TO) measured by Raman spectroscopy [34] (open square) along with the previous theoretical predictions at the $\mathrm{M}$ point (filled square) for bct-Sn are compared with the calculated phonon dispersion curve in Figure 2. The substantial difference for the LO mode may be due to the temperature and pressure differences as pointed out by Olijnyk [34]. No imaginary phonon frequencies are obtained in the phonon DOS plots for bcc-Ta, bct-Sn, $\mathrm{TaSn}_{2}, \mathrm{Ta}_{3} \mathrm{Sn}$, indicating that they are all mechanically stable at $0 \mathrm{~K}$. 

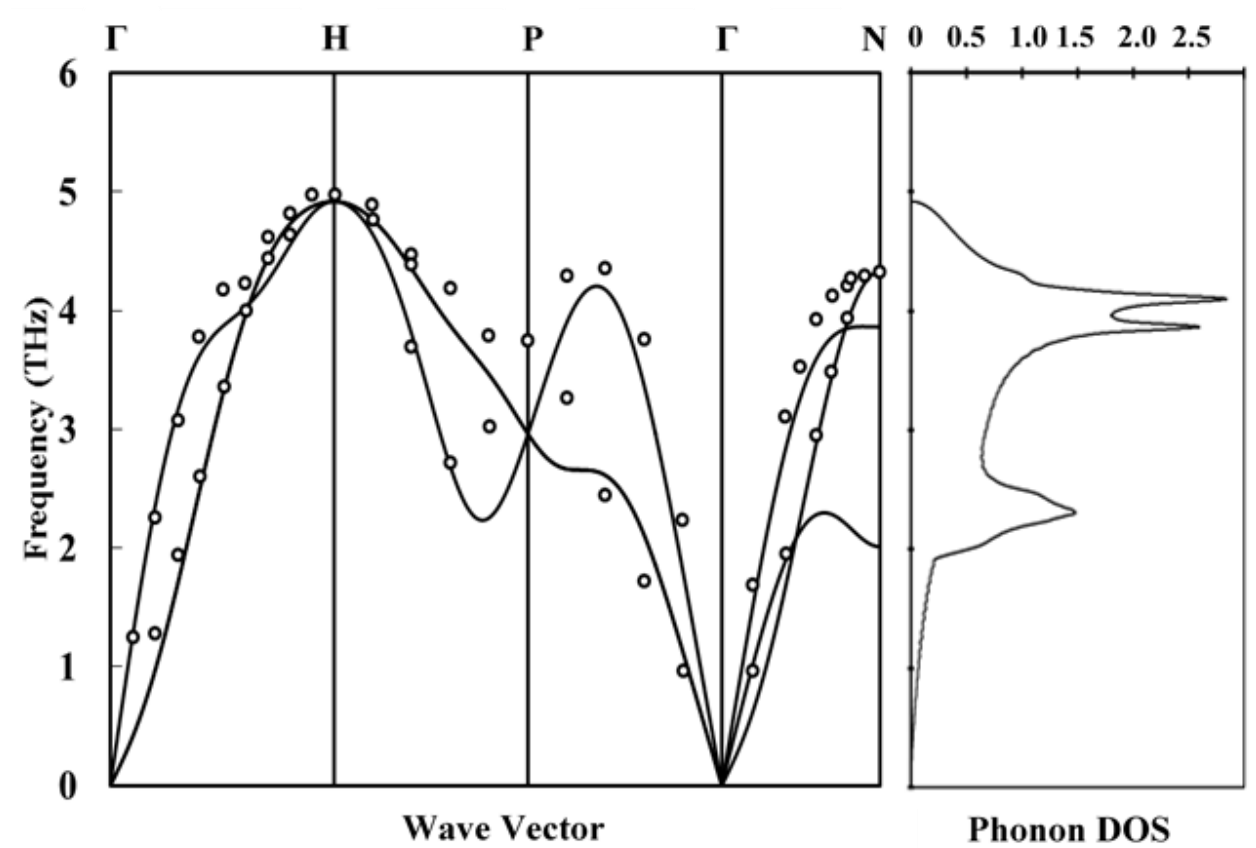

Phonon DOS

Figure 1: Calculated phonon dispersion curve of bcc-Ta, compared with neutron diffraction experiments (०) [33] along with the phonon DOS.

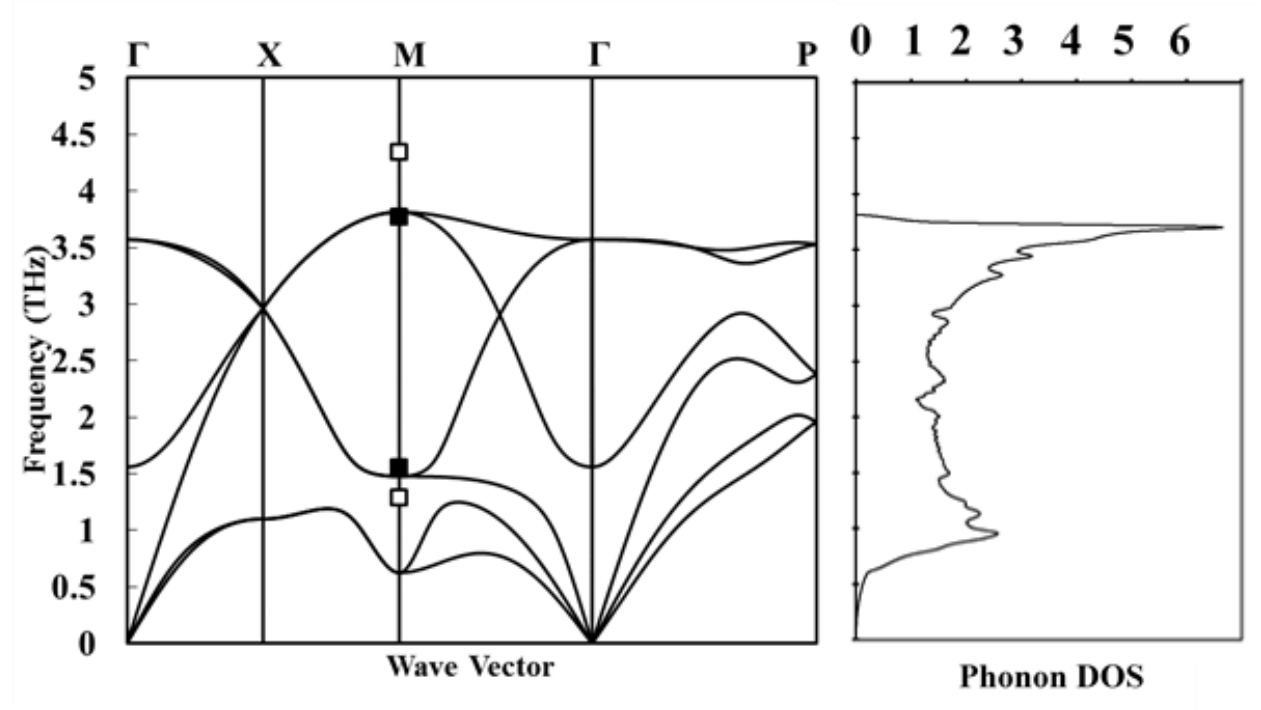

Figure 2: Calculated phonon dispersion curve of bct-Sn on the left and phonon DOS on the right. The open squares ( $\square$ ) are the LO and TO modes from Raman [34] and the filled squares the theoretical prediction of the LO and TO modes at the M point [34]. 


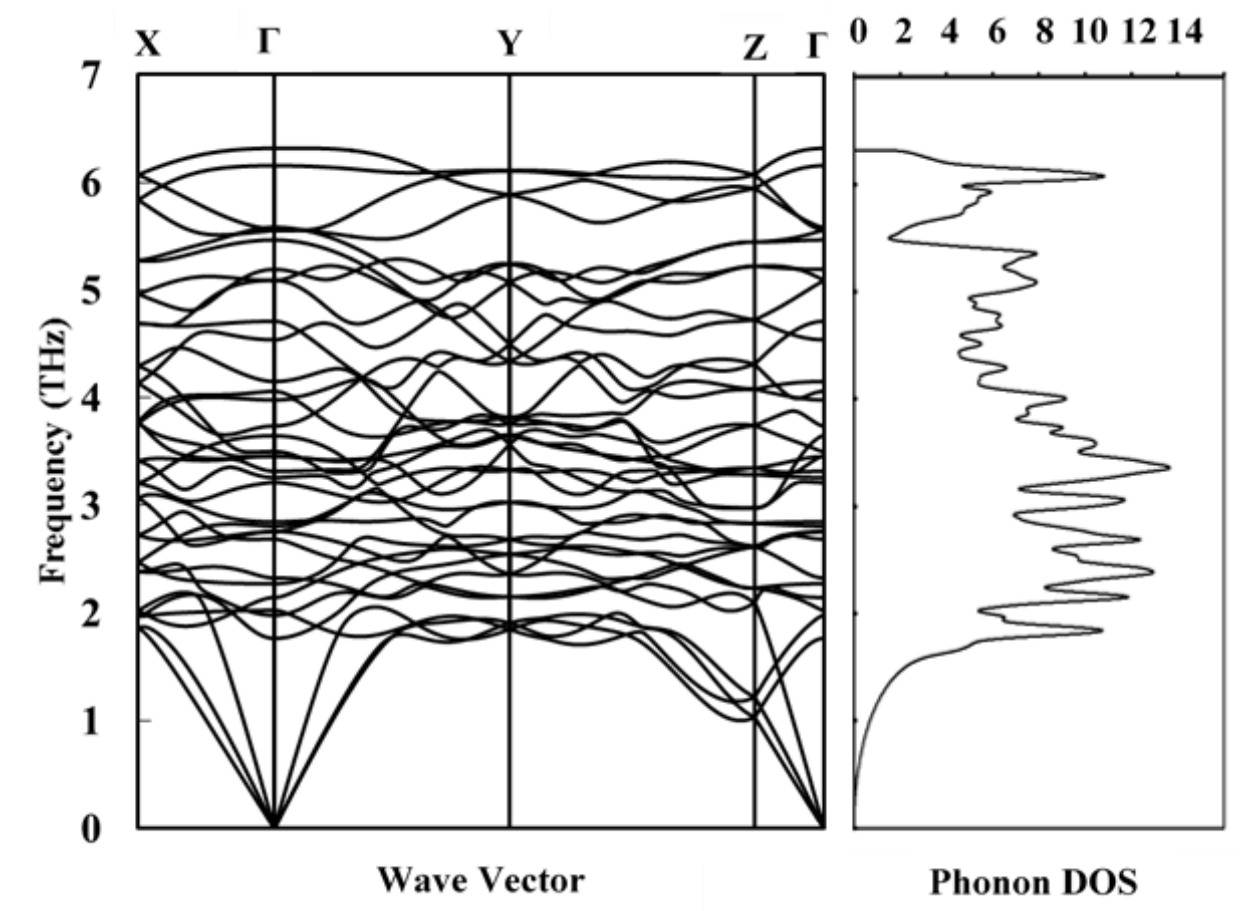

Figure 3: Calculated phonon dispersion curve for $\mathrm{TaSn}_{2}$ at $0 \mathrm{~K}$ and the phonon DOS.

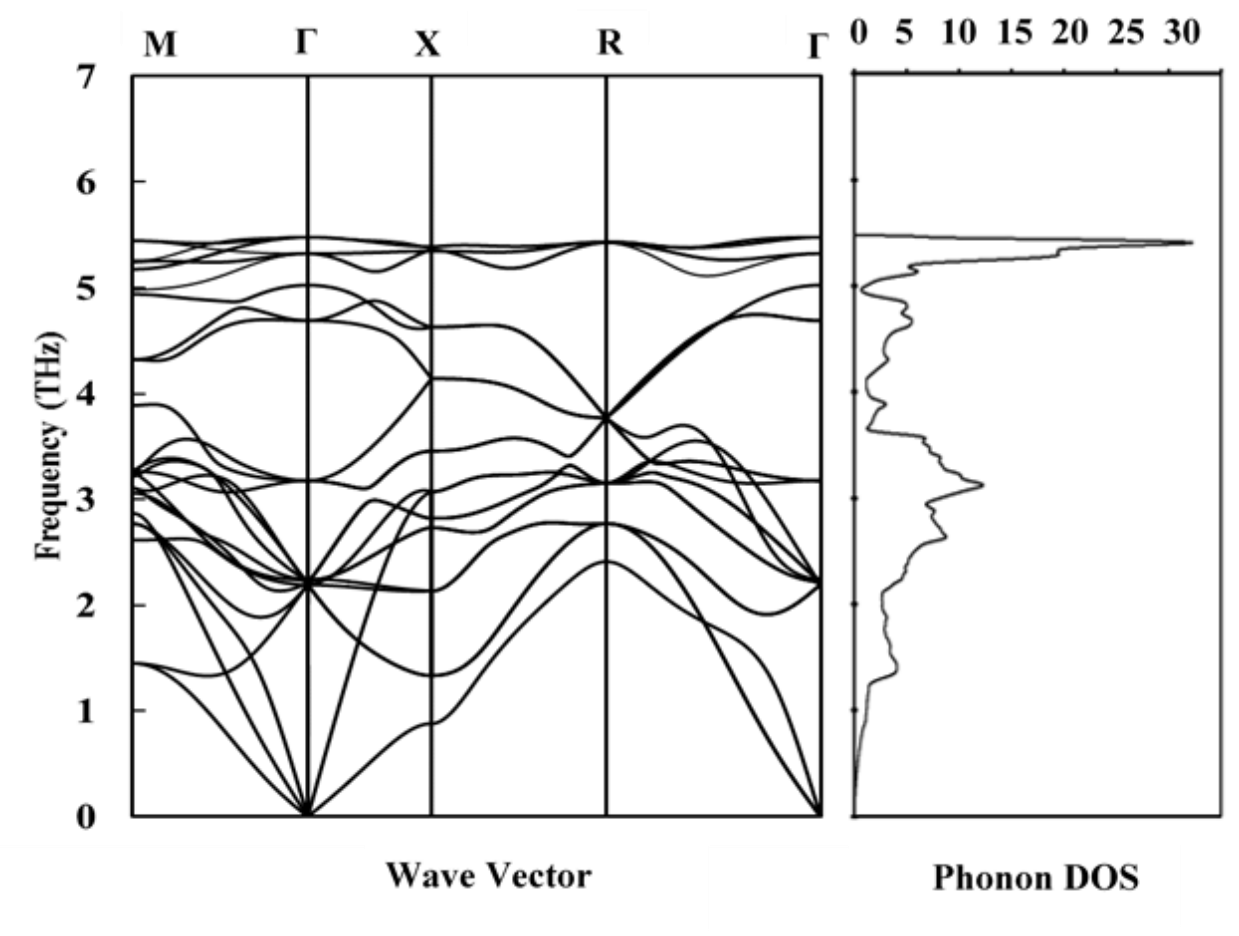

Figure 4: Calculated phonon dispersion curve of $\mathrm{Ta}_{3} \mathrm{Sn}$ at $0 \mathrm{~K}$ on the left and the phonon DOS on the right. 
The calculated lattice parameters at $0 \mathrm{~K}$ from the EOS fitting and with the Debye and phonon models at $298 \mathrm{~K}$ are compared to available experimental and previous DFT results in Table 1. The lattice parameters of Ta are compared with the experimental lattice parameters by Predmore and Arsenault [35] at room temperature and the previous 0 K DFT results by Shang et al. [36] who used the GGA-PW91 exchange correlation functional. The Sn lattice parameters are compared to experimental work by Allen et al. [37] at 298K and calculations by Arróyave et al. [38]. The properties of the $\mathrm{TaSn}_{2}$ and $\mathrm{Ta}_{3} \mathrm{Sn}$ intermetallics have not been calculated previously and are compared to experimental values by Calvert et al. [39] and Courtney et al. [17], respectively. The results show a less than $0.5 \%$ difference when compared with other DFT results at $0 \mathrm{~K}$. There is a less than $2 \%$ difference between the DFT $0 \mathrm{~K}$ results and the experiments, which are listed in Table 1. The variance is due to the fact that the calculations are at $0 \mathrm{~K}$ and the experiments are at a higher temperature. When comparing the calculated lattice parameters at $298 \mathrm{~K}$ to the experiments, all of the predictions improve to show a less than $1 \%$ difference with the exception of Sn, which shows a less than $2 \%$ difference. 
Table 1: Lattice parameters from first-principles calculations compared with experimental values.

\begin{tabular}{|c|c|c|c|c|c|}
\hline Phase & Space Group & $a(\AA)$ & $b(\AA)$ & $c(\AA)$ & Source \\
\hline \multirow[t]{5}{*}{ bcc-Ta } & $\operatorname{Im} \overline{3} \mathrm{~m}$ & 3.316 & & & This work $(0 \mathrm{~K})$ \\
\hline & & 3.328 & & & This work phonon $(298 \mathrm{~K})$ \\
\hline & & 3.330 & & & This work Debye (298 K) \\
\hline & & 3.30 & & & Expt. [35] \\
\hline & & 3.32 & & & DFT $(0 \mathrm{~K})[36]$ \\
\hline \multirow[t]{5}{*}{ bct-Sn } & I $4_{1} /$ amd & 5.939 & & 3.214 & This work $(0 \mathrm{~K})$ \\
\hline & & 5.959 & & 3.236 & This work phonon $(298 \mathrm{~K})$ \\
\hline & & 5.954 & & 3.222 & This work Debye (298 K) \\
\hline & & 5.83 & & 3.18 & Expt. [37] \\
\hline & & 5.93 & & 3.23 & DFT $(0 \mathrm{~K})[38]$ \\
\hline \multirow[t]{4}{*}{$\mathrm{TaSn}_{2}$} & Fddd & 5.641 & 9.766 & 19.200 & This work $(0 \mathrm{~K})$ \\
\hline & & 5.652 & 9.786 & 19.238 & This work phonon $(298 \mathrm{~K})$ \\
\hline & & 5.652 & 9.785 & 19.238 & This work Debye (298 K) \\
\hline & & 5.63 & 9.80 & 19.18 & Expt. [39] \\
\hline \multirow[t]{4}{*}{$\mathrm{Ta}_{3} \mathrm{Sn}$} & $\operatorname{Pm} \overline{3} \mathrm{n}$ & 5.304 & & & This work $(0 \mathrm{~K})$ \\
\hline & & 5.319 & & & This work phonon (298 K) \\
\hline & & 5.319 & & & This work Debye (298 K) \\
\hline & & 5.29 & & & Expt. [17] \\
\hline
\end{tabular}

Table 2 shows the equilibrium volume, $V$, bulk modulus, $B$, and the derivative of bulk modulus $B$ ' obtained by the EOS E-V fitting of the first-principles data at $0 \mathrm{~K}$. The $\mathrm{Sn}$ and Ta calculations are compared with previous first-principles calculations and available experiments. The volume shows a less than $0.5 \%$ difference between the previous DFT results and current DFT results for both $\mathrm{Sn}$ and $\mathrm{Ta}[35,40]$. The comparison of the DFT results at $0 \mathrm{~K}$ and the experimental results at $298 \mathrm{~K}$ for volume show a slightly higher variance of less than $5 \%$ due to the different in temperature [36,40]. The $B$ comparison of previous $0 \mathrm{~K}$ DFT results and the present $0 \mathrm{~K}$ DFT results show a less than $7 \mathrm{GPa}$ difference and the DFT results at $0 \mathrm{~K}$ vary by less than $11 \mathrm{GPa}$ from the experimental results at $298 \mathrm{~K}[35,36,40]$. The difference between the 
current calculations and the previous values may be due to many reasons; e.g. the different choices in input parameters used by Peltzer et al. [40] and different exchange correlation functionals. Another reason is due to the temperature difference $0 \mathrm{~K}$ (calculations) versus $298 \mathrm{~K}$ (experiments). Figure 5 shows the enthalpy and entropy of Ta from the Debye and phonon approaches in comparison with the data from the SGTE pure element database [29]. Figure 6 shows the comparison of the enthalpy and entropy calculated for Sn from the phonon and Debye model to the SGTE pure element database [29]. Both show excellent agreement.

Table 2: Equilibrium volume $V$, bulk modulus $B$, and the first derivative of bulk modulus with respect to pressure $B^{\prime}$, from fitted equilibrium properties from the EOS at $0 \mathrm{~K}$ compared to experimental work and previous DFT studies.

\begin{tabular}{ccccc}
\hline Phase & $V\left(\AA^{3} /\right.$ atom $)$ & $B(\mathrm{GPa})$ & $B$ & Reference \\
\hline bcc-Ta & 18.241 & 193.7 & 3.84 & This work \\
& 17.9685 & 200 & & Expt. [35] \\
& 18.313 & 195.3 & 3.82 & DFT [36] \\
bct-Sn & 28.431 & 47.7 & 4.61 & This work \\
& 27.055 & 58.0 & & Expt. [40] \\
& 28.396 & 54.0 & & DFT [40] \\
& 22.631 & 104.3 & 4.80 & This work \\
$\mathrm{TaSn}_{2}$ & 18.668 & 182.4 & 4.27 & This work \\
$\mathrm{Ta}_{3} \mathrm{Sn}$ & & & \\
\hline
\end{tabular}



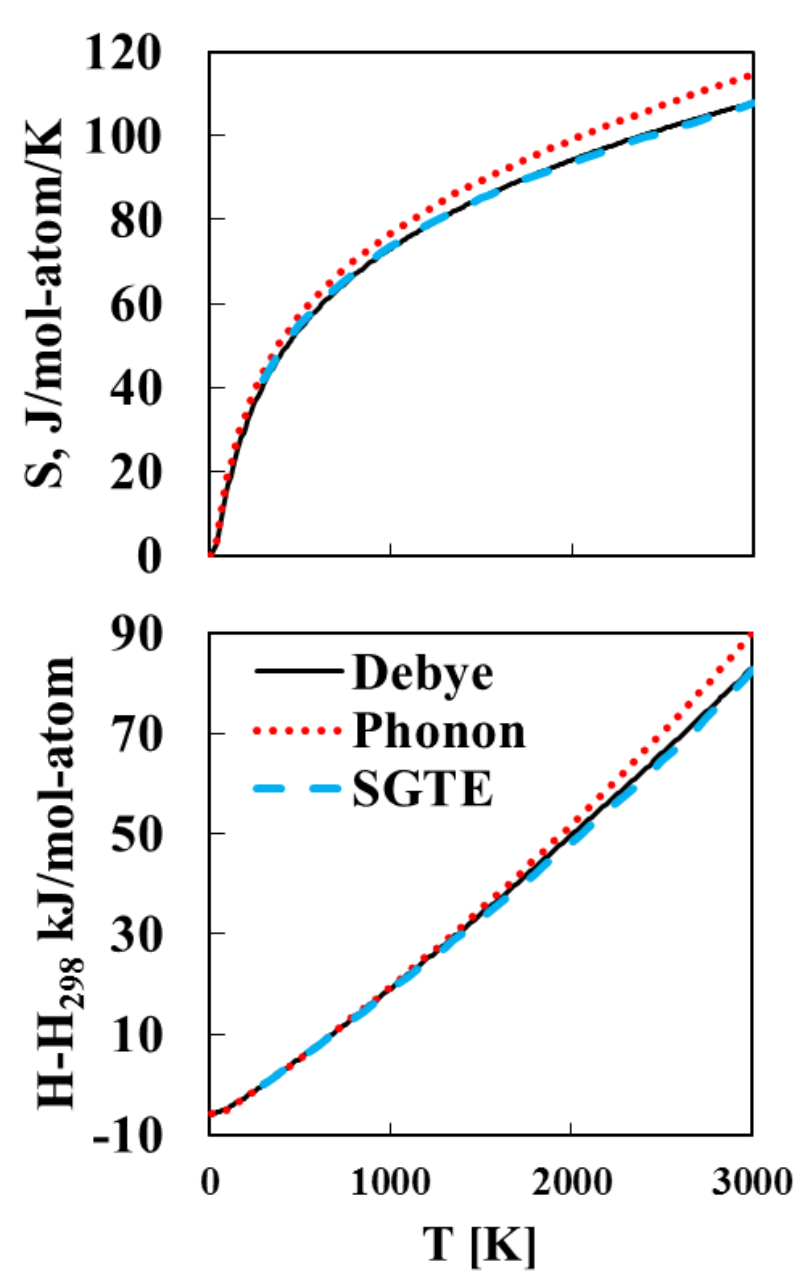

Figure 5: Comparison of the enthalpy and entropy of bcc-Ta from the Debye model (solid line) and the quasiharmonic phonon calculations (red dotted line) to the SGTE data (blue dashed line) [29] 

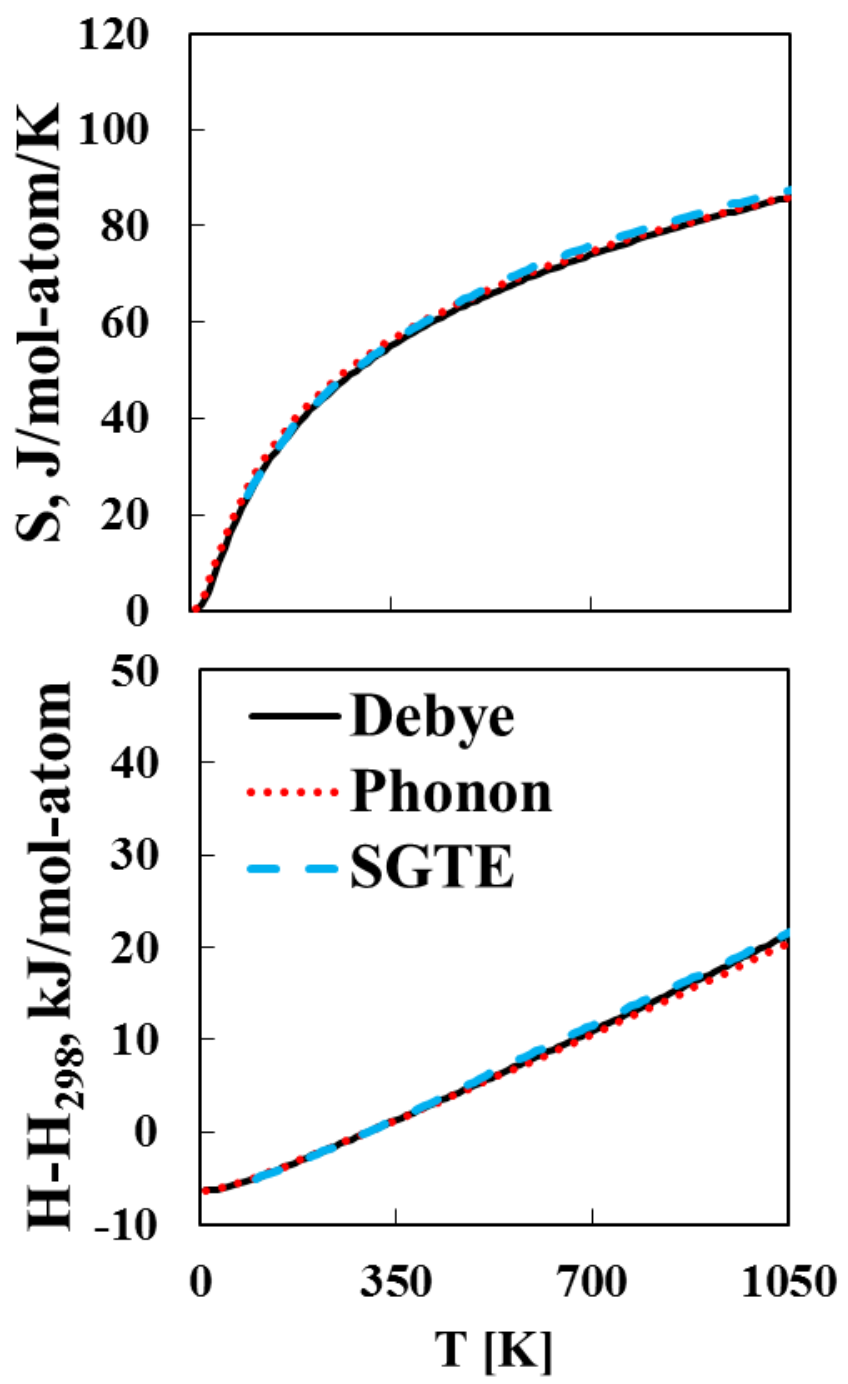

Figure 6: Comparison of the Gibbs energy of bct-Sn from the Debye model (solid line) and the quasiharmonic phonon calculations (red dotted line) to the SGTE data (blue dashed line) [29].

The elastic stiffness constants and polycrystalline elastic properties calculated by the Hill approach and the scaling factors for the Debye model are shown in Table 3. To ensure the accuracy of the scaling factor, the $B$ calculated from $c_{i j}$ is compared with the $B$ obtained from the EOS fitting, showing a difference of less than $3 \%$. Since the $B$ from the EOS fitting is already compared to experiments, the elastic calculations and the scaling factor for the Debye model are thus deemed accurate. 
Table 3: Elastic stiffness constants and elastic properties predicted using the Hill approach and the scaling factors used in the Debye model, calculated from the Poisson ratio, see Eq. 6. To ensure the accuracy of the calculated scaling factor, the bulk modulus $\left(B_{c i j}\right)$ calculated from the elastic constants was compared to the $B_{E O S}$ calculated from the EOS fitting Eq. 2.

\begin{tabular}{ccc}
\hline & $\mathrm{TaSn}_{2}$ & $\mathrm{Ta}_{3} \mathrm{Sn}$ \\
\hline $\mathrm{C}_{11}(\mathrm{GPa})$ & 166 & 313 \\
$\mathrm{C}_{12}(\mathrm{GPa})$ & 79 & 120 \\
$\mathrm{C}_{13}(\mathrm{GPa})$ & 62 & \\
$\mathrm{C}_{22}(\mathrm{GPa})$ & 189 & \\
$\mathrm{C}_{23}(\mathrm{GPa})$ & 68 & \\
$\mathrm{C}_{33}(\mathrm{GPa})$ & 187 & 71 \\
$\mathrm{C}_{44}(\mathrm{GPa})$ & 37 & \\
$\mathrm{C}_{55}(\mathrm{GPa})$ & 59 & \\
$\mathrm{C}_{66}(\mathrm{GPa})$ & 61 & 210 \\
Young's Modulus $(\mathrm{GPa})$ & 135 & 80 \\
Shear Modulus $(\mathrm{GPa})$ & 53 & 0.31 \\
Poisson Ratio & 0.288 & 0.74 \\
$\mathrm{Scaling}_{\text {factor }}$ & 0.789 & 185 \\
$\mathrm{~B}_{\text {cij }}(\mathrm{GPa})$ & 107 & 182 \\
$\mathrm{~B}_{\text {EOS }}(\mathrm{GPa})$ & 104 & \\
\hline
\end{tabular}

\subsection{CALPHAD modeling results}

The PARROT module in the Thermo-Calc software [32] is used to optimize the parameters of the Gibbs energy function of the $\mathrm{TaSn}_{2}$ and $\mathrm{Ta}_{3} \mathrm{Sn}$ intermetallics as well as the binary interaction parameters for the bcc and liquid phases. The Gibbs energy parameters of the intermetallics are first estimated from the thermodynamic properties obtained by the phonon supercell method because the phonon calculations are regarded as more accurate than the Debye model. While the decomposition temperature of the $\mathrm{TaSn}_{2}$ intermetallic is known to be $868 \mathrm{~K}$ from experiments, the decomposition of the $\mathrm{Ta}_{3} \mathrm{Sn}$ intermetallic has not been reported in the literature. It is noted that both the $\mathrm{Nb}-\mathrm{Sn}$ and $\mathrm{V}-\mathrm{Sn}$ systems, which are quite similar to the Ta-Sn system, have the $\mathrm{X}_{3} \mathrm{Sn}$ phase forming through a peritectic reaction of $\mathrm{bcc}+$ Liquid $\rightarrow \mathrm{X}_{3} \mathrm{Sn}[16,14]$. Based on the

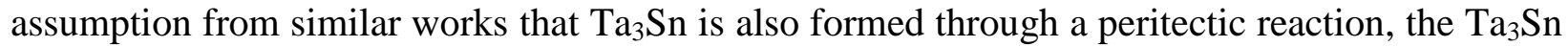
parameters are adjusted and the parameters for the liquid phase are evaluated. The evaluation of 
the Gibbs parameters along with the results from the Debye model and the phonon quasiharmonic approach for $\mathrm{TaSn}_{2}$ and $\mathrm{Ta}_{3} \mathrm{Sn}$ are plotted in Figure 7 and Figure 8, respectively. As seen in both figures, the data from the phonon method correlates well with the current CALPHAD modeling. This is to be expected since this data was used to evaluate the parameters. It is noted in Figure 7, that the heat capacity and entropy of $\mathrm{TaSn}_{2}$ from the current CALPHAD modeling is higher than those from the first-principles calculations. This is due to the fact that the enthalpy and entropy values from DFT were adjusted with the experimental data of the peritectic temperature. 

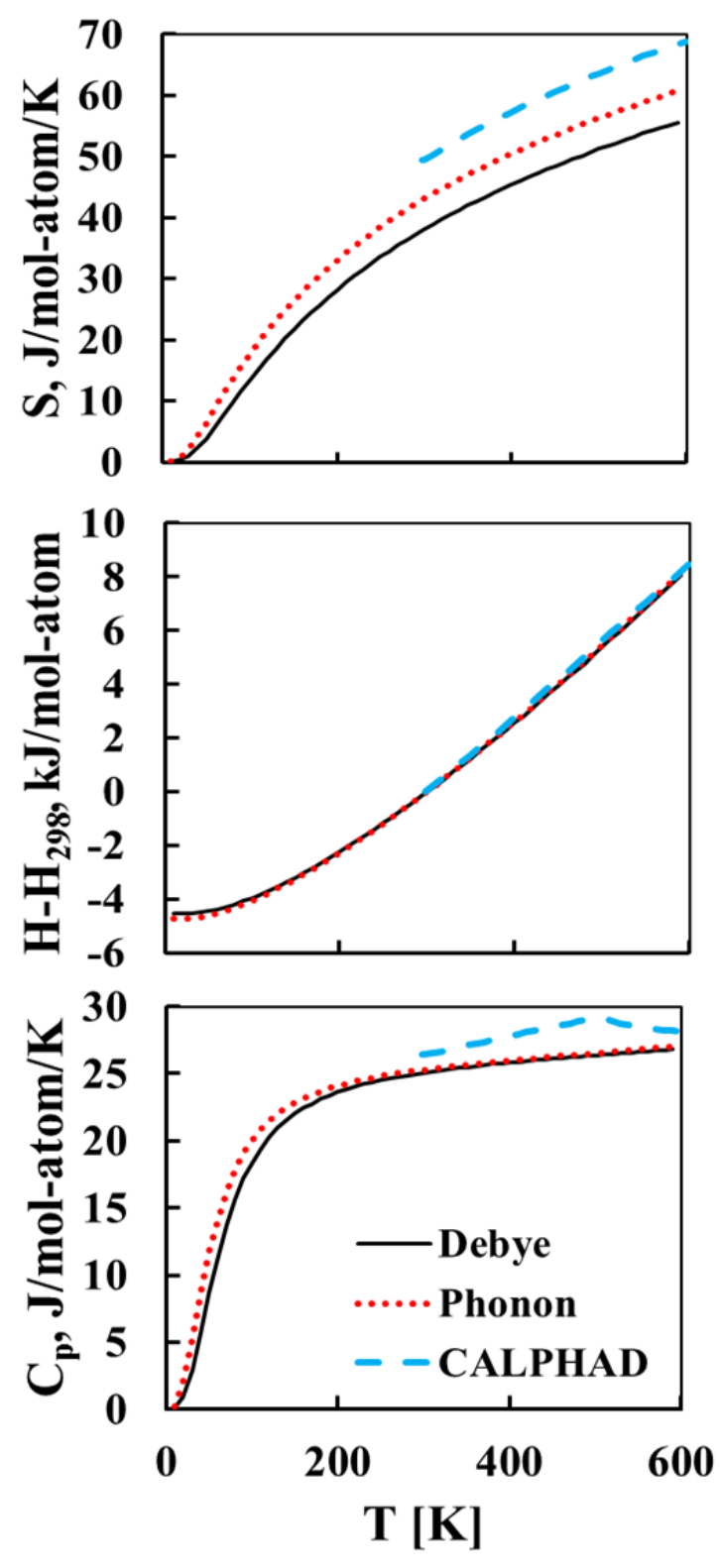

Figure 7: Heat capacity, enthalpy and entropy of $\mathrm{TaSn}_{2}$ using the Debye model (solid line) and the quasiharmonic phonon calculation (red dotted line) from first-principles calculations, compared with those from the current CALPHAD modeling (blue dashed line). 

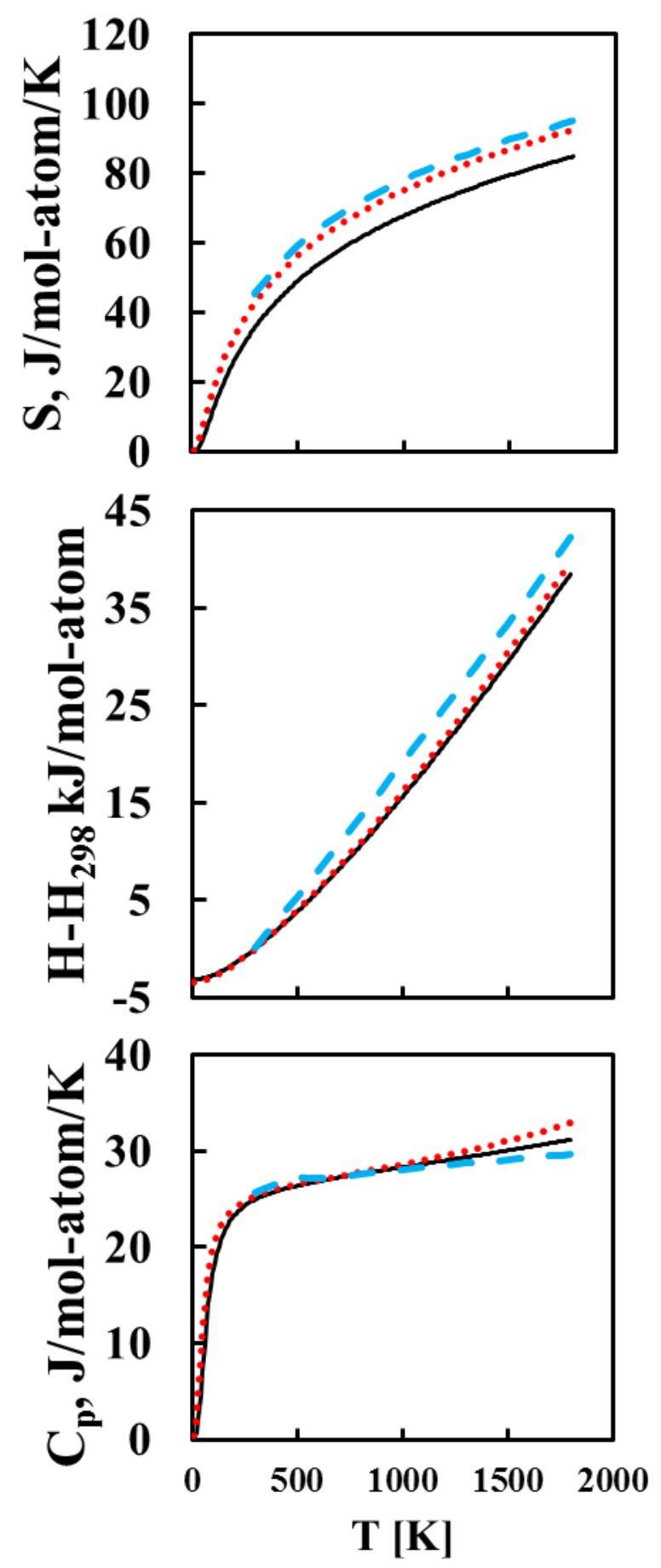

Figure 8: Heat capacity, enthalpy and entropy of $\mathrm{Ta}_{3} \mathrm{Sn}$ using the Debye model (solid line) and the quasiharmonic phonon calculation (red dotted line) compared with those from the current CALPHAD modeling (blue dashed line).

The bct and diamond phases are treated as ideal due to the little solubility. As previously stated, the enthalpies of formation of the bcc phase for five different Sn-Ta compositions are calculated 
and plotted in Figure 9, showing asymmetrical behavior. There is a discrepancy between the first-principles value and the CALPHAD modeling for the lattice stability of bcc-Sn. The firstprinciples predicts a value of $15.48 \mathrm{~kJ} / \mathrm{mol}$-atom and the CALPHAD model gives $4.42 \mathrm{~kJ} / \mathrm{mol}$ atom. This difference is expected to be due to the instability of Sn in the bcc phase. Wang et al. [41] concluded and discussed the same discrepancy when comparing first-principles DFT results to SGTE data for Os and $\mathrm{Ru}$. Wang et al. calculated the lattice stability of bec and fec structure for $\mathrm{Os}$ and $\mathrm{Ru}$, both stable in the hexagonal cubic phase at standard temperature and pressure, and concluded a difference of approximately 40 and $60 \mathrm{~kJ} / \mathrm{mol}$ for $\mathrm{Ru}$ and Os, respectively. Wang et al. attributed this difference to the fact that when using first-principles calculations of unstable structures, frequencies of some of the phonon modes would become imaginary and thus the results would be less accurate. On the other hand, the CALPHAD technique can extrapolate lattice stabilities from binary solutions for which an alloying element has stabilized the otherwise unstable structure. These enthalpies of formation calculated from the SQS first-principles calculations are used to evaluate the bcc binary interaction parameters in the present CALPHAD modeling. The enthalpy of formation of the bcc phase is negative at the Ta rich side and becomes positive at the Sn rich side. This is common for X-Sn systems [16,14], such as the Nb-Sn system [16] shown in Figure 9. It should be noted that Toffolon et al. [16,15] used experimental data on the $\mathrm{Sn}$-rich bcc phase to evaluate the $\mathrm{Nb}-\mathrm{Sn}$ system's bcc interaction parameters. Due to the asymmetry of enthalpy of formation for the bcc phase, a subregular ${ }^{1} \mathrm{~L}$ interaction parameters is introduced. 


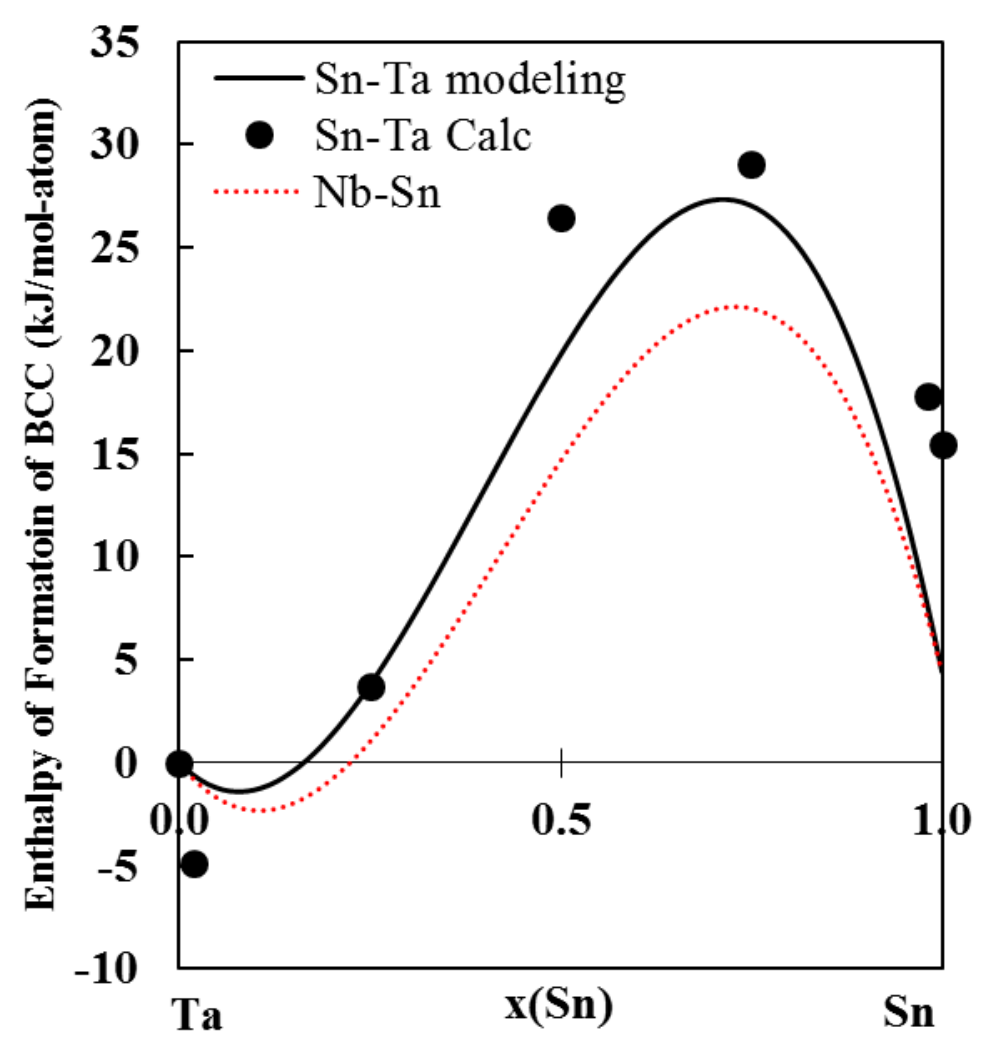

Figure 9: Enthalpy of formation of the bcc phase of the Sn-Ta system as a function of composition at 298 $\mathrm{K}$ and ambient pressure from the current CALPHAD modeling (solid line) and from the first-principles calculations (dots), showing asymmetric behavior. This was compared with data of the Nb-Sn system from Toffolon et al.[16] (dashed red line) which was modeled using experimental data, showing similar asymmetric behavior.

The interaction parameters obtained in the present work are listed in Table 4. Based on these model parameters, the phase diagram is calculated and shown in Figure 10. The melting temperature of $\mathrm{Ta}_{3} \mathrm{Sn}$ is predicted to be $2884 \mathrm{~K}$. Both the intermetallics decompose incongruently similar to those in the $\mathrm{Nb}-\mathrm{Sn}$ and V-Sn systems. As seen in Table 4, both intermetallic phases have a negative enthalpy of formation and a negative entropy of formation. This goes along with previous predictions by Arroyave and Liu [42] where they showed that the enthalpy and entropy of formation have the same sign. The calculated enthalpy of mixing of the liquid phase is plotted in Figure 11. The interaction parameter for the liquid phase allows for an accurate representation of the phase stability in Figure 10 but may need to be slightly adjusted if experimental data would come available. 
Table 4: Modeled parameters in SI units in the present work for the phases in the Sn-Ta binary system. These parameters were incorporated with the SGTE data for the pure elements [29].

\begin{tabular}{|c|c|}
\hline Phase (model) & Modeled Parameters \\
\hline bcc_A2 (Sn, Ta) & $\begin{array}{c}{ }^{0} L_{T a, S n}^{b c c}=+70451 \\
{ }^{1} L_{T a, S n}^{b c c}=+112237\end{array}$ \\
\hline Liquid (Sn, Ta) & ${ }^{0} L_{T a, S n}^{L i q}=-17118$ \\
\hline $\mathrm{TaSn}_{2}$ & $G^{T^{T a S n_{2}}}=2^{o} G_{S n}^{b c t}+{ }^{o} G_{T a}^{b c c}-29678-4.202 T$ \\
\hline $\mathrm{Ta}_{3} \mathrm{Sn}$ & $G^{T a_{3} S n}={ }^{o} G_{S n}^{b c t}+3^{o} G_{T a}^{b c c}-68844-6.000 T$ \\
\hline
\end{tabular}

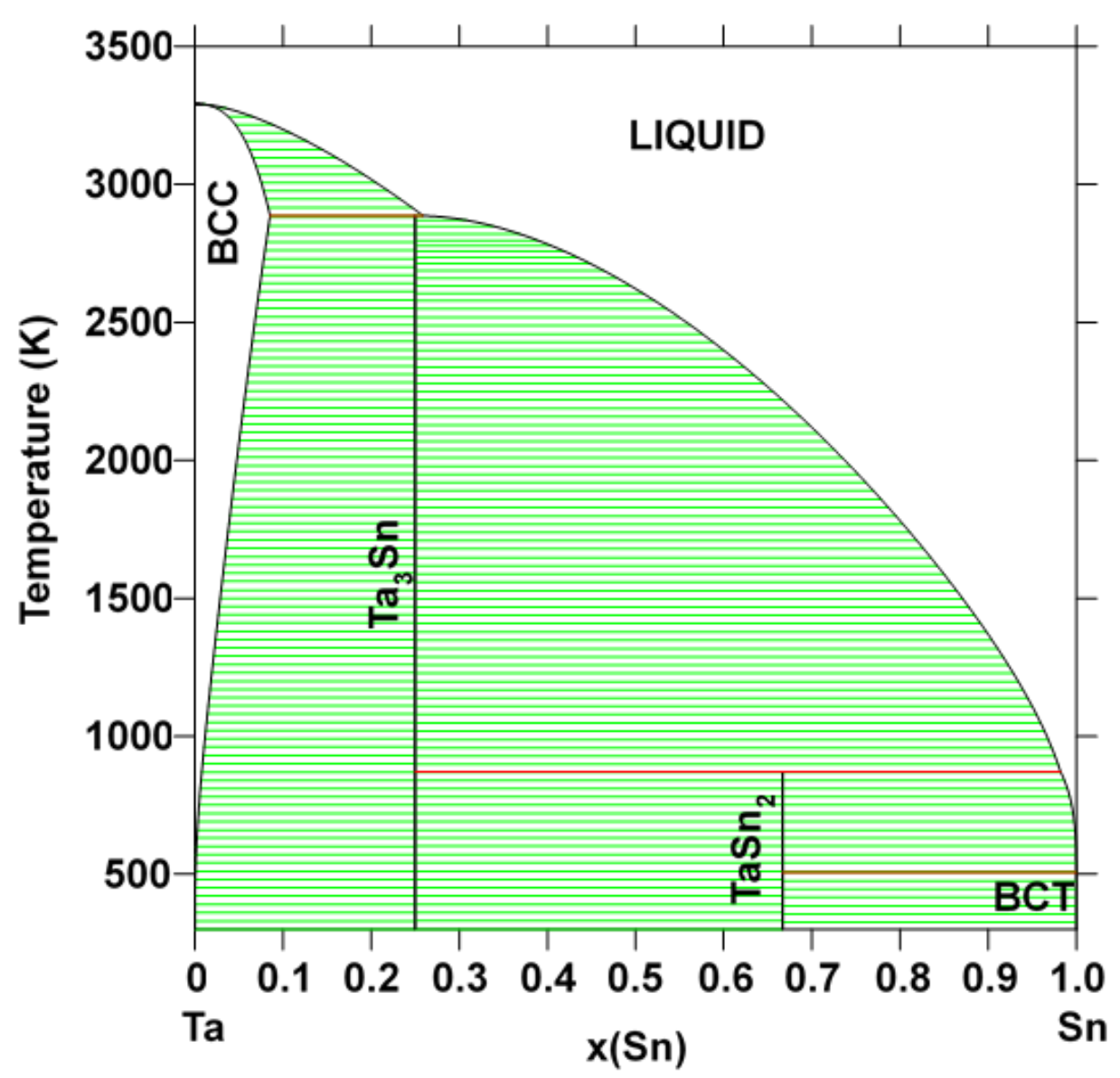

Figure 10: Calculated Sn-Ta phase diagram using the present thermodynamic description. 


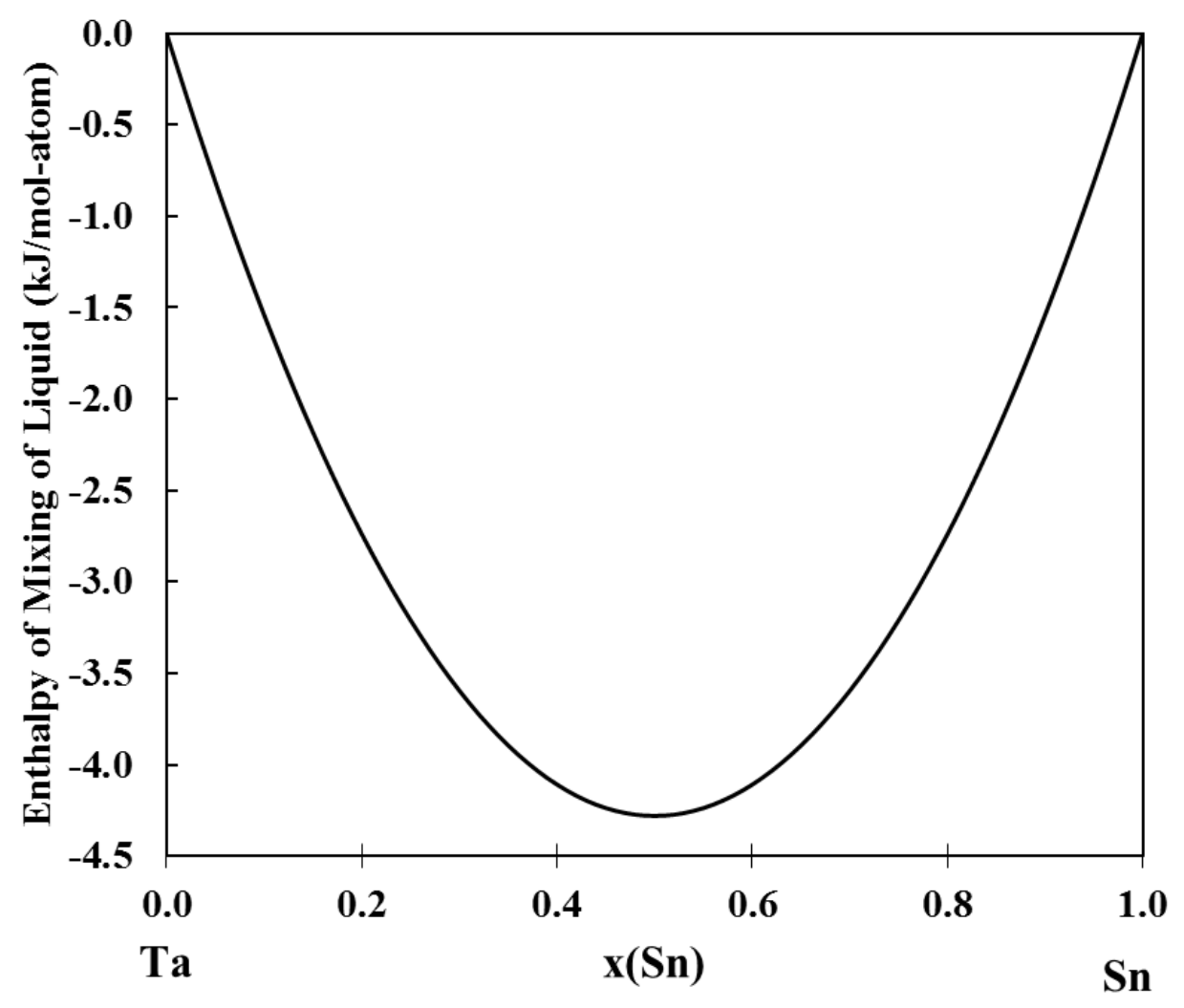

Figure 11: Enthalpy of mixing of the liquid phase as a function of composition at $298 \mathrm{~K}$ and ambient pressure in the Sn-Ta system.

\section{Conclusion}

The present work incorporates the thermodynamic data from DFT-based first-principles calculations and the available experimental data in the literature to model the Gibbs energies for the bcc and liquid solution phases and the stoichiometric $\mathrm{Ta}_{3} \mathrm{Sn}$ and $\mathrm{TaSn}_{2}$ phases of the $\mathrm{Sn}-\mathrm{Ta}$ system. First-principles calculations are used to predict the enthalpy of mixing of the bcc phase for the evaluation of interaction parameters in the phase. The decomposition temperature of $\mathrm{Ta}_{3} \mathrm{Sn}$ is predicted to be $2884 \mathrm{~K}$.

\section{Acknowledgements}


This work is funded by the National Science Foundation (NSF) with Grant No. CMMI-1333999. First-principles calculations were carried out on the LION Clusters at the Pennsylvania State University supported by the Materials Simulation Center and the Institute of Computational Science. Calculations were also carried out on the CyberStar cluster funded by the NSF through Grant No. OCI-0821527 and the Extreme Science and Engineering Discovery Environment 9 (XSEDE) which is supported by NSF Grant No. ACI-1053575. We would also like to thank the members of the Phases Research Lab at Penn State for stimulating discussion and for the critical reading of the manuscript. 
References:

[1] M. Long, H.J. Rack, Titanium alloys in total joint replacement - a materials science perspective, Biomaterials. 19 (1998) 1621-1639. doi:10.1016/S0142-9612(97)00146-4.

[2] M. Geetha, A.K. Singh, R. Asokamani, A.K. Gogia, Ti based biomaterials, the ultimate choice for orthopaedic implants - A review, Prog. Mater. Sci. 54 (2009) 397-425. doi:10.1016/j.pmatsci.2008.06.004.

[3] V. Brailovski, S. Prokoshkin, M. Gauthier, K. Inaekyan, S. Dubinskiy, M. Petrzhik, et al., Bulk and porous metastable beta $\mathrm{Ti}-\mathrm{Nb}-\mathrm{Zr}$ (Ta) alloys for biomedical applications, Mater. Sci. Eng. C. 31 (2011) 643-657.

[4] M. Niinomi, M. Nakai, J. Hieda, Development of new metallic alloys for biomedical applications, Acta Biomater. 8 (2012) 3888-3903.

[5] D. Kuroda, M. Niinomi, M. Morinaga, Y. Kato, T. Yashiro, Design and mechanical properties of new $\beta$ type titanium alloys for implant materials, Mater. Sci. Eng. A. 243 (1998) 244-249.

[6] G. He, M. Hagiwara, Ti-Cu-Ni (Fe, Cr, Co)-Sn-Ta (Nb) alloys with potential for biomedical applications, Mater. Trans. 45 (2004) 1120-1123.

[7] G. He, M. Hagiwara, Ti alloy design strategy for biomedical applications, Mater. Sci. Eng. C. 26 (2006) 14-19. doi:10.1016/j.msec.2005.03.007.

[8] H. Okamoto, Sn-Ta (Tin-Tantalum), J. Phase Equilibria. 24 (2003) 484.

[9] Z.-K. Liu, First-Principles Calculations and CALPHAD Modeling of Thermodynamics, J. Phase Equilibria Diffus. 30 (2009) 517-534.

[10] S.L. Shang, Y. Wang, D.E. Kim, Z.-K. Liu, First-principles thermodynamics from phonon and Debye model: Application to Ni and Ni3Al, Comput. Mater. Sci. 47 (2010) 1040-1048.

[11] A. Wang, S. Shang, D. Zhao, J. Wang, L. Chen, Y. Du, et al., Structural, phonon and thermodynamic properties of fcc-based metal nitrides from first-principles calculations, Calphad. 37 (2012) 126-131. doi:10.1016/j.calphad.2012.02.008.

[12] T. Studnitzky, R. Schmid-Fetzer, Phase Formation and reaction Kinetics in M-Sn systems (M=Zr, Hf, Nb, Ta, Mo), Zeitschrift Fur Met. 93 (2002) 894-903.

[13] F. BASILE, CRISTALLOGRAPHIC STUDY AND SUPRACONDUCTIVE PROPERTIES OF COMPOUNDS V3SN AND TA2SN3, in: Ann. Chim. Fr., 1971: p. 241-\&.

[14] Q. Yue, Y.Q. Liu, M.Y. Chu, J.Y. Shen, Thermodynamic modeling of the Sn-V binary system, Calphad. 33 (2009) 539-544.

[15] C. Toffolon, C. Servant, B. Sundman, Thermodynamic assessment of the Nb-Sn system, J. Phase Equilibria. 19 (1998) 479-485. doi:10.1361/105497198770341978.

[16] C. Toffolon, C. Servant, J.C. Gachon, B. Sundman, Reassessment of the Nb-Sn system, J. 
Phase Equilibria. 23 (2002) 134-139.

[17] T.H. Courtney, G.W. Pearsall, J. Wulff, Effect of Processing History on the Superconducting Properties and Long-Range Order of Ta3Sn, J. Appl. Phys. 36 (1965) 3256-3260.

[18] Y. Wang, Z.-K. Liu, L.-Q. Chen, Thermodynamic properties of Al, Ni, NiAl, and Ni3Al from first-principles calculations, Acta Mater. 52 (2004) 2665-2671.

doi:10.1016/j.actamat.2004.02.014.

[19] V.L. Moruzzi, J.F. Janak, K. Schwarz, Calculated thermal properties of metals, Phys. Rev. B. 37 (1988) 790-799. doi:10.1103/PhysRevB.37.790.

[20] X.L. Liu, B.K. VanLeeuwen, S.-L. Shang, Y. Du, Z.-K. Liu, On the scaling factor in Debye-Grüneisen model: A case study of the $\mathrm{Mg}-\mathrm{Zn}$ binary system, Comput. Mater. Sci. 98 (2015) 34-41. doi:10.1016/j.commatsci.2014.10.056.

[21] G. Simmons, H. Wang, Single crystal elastic constants and calculated aggregate properties: A handbook, M.I.T. Press, Cambridge, 1971.

[22] S.L. Shang, Y. Wang, Z.-K. Liu, First-principles elastic constants of alpha- and thetaAl2O3, Appl. Phys. Lett. 90 (2007) 101909. doi:10.1063/1.2711762.

[23] C. Jiang, C. Wolverton, J. Sofo, L.-Q. Chen, Z.-K. Liu, First-principles study of binary bcc alloys using special quasirandom structures, Phys. Rev. B. 69 (2004) 214202. doi:10.1103/PhysRevB.69.214202.

[24] G. Kresse, J. Furthmüller, Efficiency of ab-initio total energy calculations for metals and semiconductors using a plane-wave basis set, Comput. Mater. Sci. 6 (1996) 15-50.

[25] G. Kresse, D. Joubert, From ultrasoft pseudopotentials to the projector augmented-wave method, Phys. Rev. B. 59 (1999) 1758-1775.

[26] P.E. Blöchl, Projector augmented-wave method, Phys. Rev. B. 50 (1994) 17953.

[27] J.P. Perdew, K. Burke, M. Ernzerhof, Generalized gradient approximation made simple, Phys. Rev. Lett. 77 (1996) 3865.

[28] H.J. Monkhorst, J.D. Pack, Special points for Brillouin-zone integrations, Phys. Rev. B. 13 (1976) 5188-5192.

[29] A.T. Dinsdale, SGTE Data for Pure Elements, CALPHAD. 15 (1991) 317-425.

[30] O. Redlich, A.T. Kister, Algebraic Representation of Thermodynamic Properties and the Classification of Solutions, Ind. Eng. Chem. 40 (1948) 345-348. doi:10.1021/ie50458a036.

[31] M. Hillert, The compound energy formalism, J. Alloys Compd. 320 (2001) 161-176.

[32] J.-O. Andersson, T. Helander, L. Höglund, P. Shi, B. Sundman, Thermo-Calc \&amp; DICTRA, computational tools for materials science, Calphad. 26 (2002) 273-312. doi:10.1016/S0364-5916(02)00037-8.

[33] S. Taioli, C. Cazorla, M.J. Gillan, D. Alfè, Melting curve of tantalum from first principles, Phys. Rev. B. 75 (2007) 214103. doi:10.1103/PhysRevB.75.214103. 
[34] H. Olijnyk, Pressure dependence of Raman phonons of metallic $\beta$-Sn, Phys. Rev. B. 46 (1992).

[35] R. Predmore, R.J. Arsenault, Short range order of Ta-Mo B.C.C. alloys, Scr. Metall. 4 (1970) 213-217. doi:10.1016/0036-9748(70)90195-X.

[36] S.L. Shang, A. Saengdeejing, Z.G. Mei, D.E. Kim, H. Zhang, S. Ganeshan, et al., Firstprinciples calculations of pure elements: Equations of state and elastic stiffness constants, Comput. Mater. Sci. 48 (2010) 813-826. doi:10.1016/j.commatsci.2010.03.041.

[37] W.P. Allen, J.H. Perepezko, Solidification of undercooled Sn-Sb peritectic alloys: Part I. Microstructural evolution, Metall. Trans. A. 22 (1991) 753-764. doi:10.1007/bf02670298.

[38] R. Arroyave, Z.-K. Liu, Intermetallics in the Mg-Ca-Sn ternary system: Structural, vibrational, and thermodynamic properties from first principles, Phys. Rev. B. 74 (2006).

[39] L.D. Calvert, P. Villars, Pearson's Handbook of Crystallographic Data for Intermetallic Phases, ASM, Mater. Park. OH. (1991).

[40] E.L. Peltzer y Blancá, A. Svane, N.E. Christensen, C.O. Rodríguez, O.M. Cappannini, M.S. Moreno, Calculated static and dynamic properties of $\beta-\mathrm{Sn}$ and $\mathrm{Sn}-\mathrm{O}$ compounds, Phys. Rev. B. 48 (1993) 15712-15718. doi:10.1103/PhysRevB.48.15712.

[41] Y. Wang, S. Curtarolo, C. Jiang, R. Arroyave, T. Wang, G. Ceder, et al., Ab initio lattice stability in comparison with CALPHAD lattice stability, Calphad. 28 (2004) 79-90.

[42] R. Arroyave, Z.K. Liu, Thermodynamic modelling of the Zn-Zr system, Calphad. 30 (2006) 1-13. 